Research Article

\title{
Locating-Total Domination Number of Cacti Graphs
}

\author{
Jianxin Wei $\mathbb{D}^{1},{ }^{1}$ Uzma Ahmad, $^{2}$ Saira Hameed, ${ }^{2}$ and Javaria Hanif ${ }^{2}$ \\ ${ }^{1}$ School of Mathematics and Statistics Science, Ludong University, Yantai, Shandong 264025, China \\ ${ }^{2}$ Department of Mathematics, University of the Punjab, Lahore, Pakistan
}

Correspondence should be addressed to Jianxin Wei; wjx0426@ldu.edu.cn

Received 13 August 2020; Revised 7 September 2020; Accepted 30 September 2020; Published 19 October 2020

Academic Editor: M. Javaid

Copyright (C) 2020 Jianxin Wei et al. This is an open access article distributed under the Creative Commons Attribution License, which permits unrestricted use, distribution, and reproduction in any medium, provided the original work is properly cited.

For a connected graph $J$, a subset $W \subseteq V(J)$ is termed as a locating-total dominating set if for $a \in V(J), N(a) \cap W \neq \phi$, and for $a, b \in V(J)-W, N(a) \cap W \neq N(b) \cap W$. The number of elements in a smallest such subset is termed as the locating-total domination number of $J$. In this paper, the locating-total domination number of unicyclic graphs and bicyclic graphs are studied and their bounds are presented. Then, by using these bounds, an upper bound for cacti graphs in terms of their order and number of cycles is estimated. Moreover, the exact values of this domination variant for some families of cacti graphs including tadpole graphs and rooted products are also determined.

\section{Introduction}

Let $J=(V, E)$ be a simple undirected and connected graph with vertex set $V=V(J)$ and edge set $E=E(J)$. The number of vertices in $J$ is called the order of $J$. The set of vertices in $V$ adjacent to any vertex $v$ is called the open neighbourhood of $v$ in $V$, denoted by $N_{J}(v)$, and the set $N_{J}(v) \bigcup\{v\}$ is called the closed neighbourhood of $v$ in $V$, denoted by $N_{J}[v]$. A vertex of degree one is called leaf (pendant) and its unique neighbouring vertex is called support vertex. A set $W \subseteq V(J)$ is said to be a dominating set of $J$ if every vertex in $V-W$ has nonempty open neighbourhood in $W$ and furthermore if $N(a) \cap W \neq \phi$ for every vertex $a \in V(J)$, then this set $W$ is referred to as a total dominating set in $J$. A total dominating set $W$ with the additional condition that every pair of distinct vertices outside $W$ have distinct open neighbourhood in $W$ is known as a locating-total dominating set, abbreviated as LTD-set. The smallest dominating, total dominating, and locating-total dominating sets of $J$ are denoted by $\gamma(J)$-set, $\gamma_{t}(J)$-set, and $\gamma_{t}^{L}(J)$-set, respectively, and their cardinalities are known as domination, total domination, and locating-total domination numbers, respectively.

A path and cycle having $m$ vertices are denoted by $P_{m}$ and $C_{m}$. A simple connected graph having no cycle is a tree.
A graph is a unicyclic or bicyclic graph if it contains exactly one cycle or two cycles, respectively. A graph in which any two distinct cycles have maximum one vertex in common is termed as a cactus. A tadpole graph is obtained by joining a leaf (pendant) vertex of path to a vertex of a cycle through an edge. For connected graphs $K$ and $L$, the rooted product of $K$ by $L(K \odot L)$ is obtained by considering $|K|$ copies of $L$ and identifying the $i$-th vertex of $L$ with the root vertex of $i$-th copy of $L$. The idea of locating-total domination and differentiating-total domination was presented first by Haynes et al. [1]. They studied these variants of domination for trees and estimated a bound on this class. The differentiating-total and locating-total domination numbers of path graphs are also determined. The improved new bound for locating-total dominating number of trees is presented by Chen and Sohn [2]. They also gave the sharpness of these bounds and identified the trees achieving these bounds. The different versions of locating-total domination number for different classes of graphs are investigated in [3-5]. The bounds on locating-total domination number for different types of products are studied in $[6,7]$. A new bound of locating-total domination number using annihilation number of a tree was presented by Ning et al. [8]. They also identified the trees attaining this bound. In this paper, locating-total domination number of unicyclic and bicyclic graphs is investigated 
and their upper bounds are given. Consequently, using these bounds, an upper bound for cacti graphs is presented. Moreover, the exact values of this domination variant for some classes of cacti graphs are computed. In particular tadpole graphs, rooted products of path by cycle graph and cycle by path graph are computed.

\section{Bounds of Locating-Total Domination Number of Cacti Graphs}

Theorem 1 (see [2]). For a tree $\mathfrak{\Im}$ having $r \geq 3$ vertices and $l$ pendant vertices, one has

$$
\gamma_{t}^{L}(\Im) \leq \frac{r+l}{2}
$$

Proposition 1 (see [9]). The $\gamma_{t}^{L}$-set of a graph J must include all the support vertices of $J$.

A unicycle graph $U$ having $r \geq 3$ vertices can be obtained by connecting any two nonadjacent vertices of a tree $\mathfrak{\Im}$ having $r \geq 3$ vertices by an edge.

Lemma 1. Let $W$ be a $\gamma_{t}^{L}$-set of a tree $\Im$ with order $r \geq 4$ and $l^{\prime}$ pendant vertices. Let $U$ be the unicyclic graph of order $r \geq 4$ with $l$ pendant vertices constructed by joining any two nonadjacent vertices $u, v \in V(\mathfrak{I})$ through an edge. If either both belong to $W$ or none of them belongs to $W$, then

$$
\gamma_{t}^{L}(U) \leq \frac{r+l+2}{2} .
$$

Proof. It is easy to see that the $\gamma_{t}^{L}$-set of a graph $G^{\prime}$ obtained by adding edges between two vertices of $\gamma_{t}^{L}$-set or between two vertices not in $\gamma_{t}^{L}$-set of a graph $G$ is the same as that of $\gamma_{t}^{L}$-set of $G$. This implies that $W$ also becomes a $\gamma_{t}^{L}$-set for $U$. Hence, by Theorem 1, we have

$$
\gamma_{t}^{L}(U)=|W|=\gamma_{t}^{L}(\mathfrak{\Im}) \leq \frac{r+l^{\prime}}{2} .
$$

Two cases arise:

Case 1: Assume that one of them is a pendant vertex. If exactly one is a pendant vertex, then $l=l^{\prime}-1$ and by (3),

$$
\gamma_{t}^{L}(U) \leq \frac{r+l+1}{2}<\frac{r+l+2}{2} .
$$

But if both are leaves, then $l=l^{\prime}-2$ and by (3),

$$
\gamma_{t}^{L}(U) \leq \frac{r+l+2}{2}
$$

Case 2: Now assume none of them is a leaf; then, $l^{\prime}=l$ and by (3),

$$
\gamma_{t}^{L}(U) \leq \frac{r+l}{2}<\frac{r+l+2}{2} .
$$

Theorem 2. Let $U$ be a unicycle graph having $r \geq 3$ vertices and $l$ pendant vertices. Then,

$$
\gamma_{t}^{L}(U) \leq \frac{r+l+3}{2} .
$$

Proof. Let $\mathfrak{I}$ be a tree of order $r$ with $l$ ' leaves and $u, v \in V(\mathfrak{I})$ be its two nonadjacent vertices such that unicycle $U$ is obtained from $\mathfrak{\Im}$ by joining $u$ and $v$. Let $W$ be a $\gamma_{t}^{L}$-set of $\mathfrak{\Im}$. Now the vertices $u$ and $v$ may belong to $W$ or not. If both vertices $u$ and $v$ belong to $W$ or both vertices $u$ and $v$ do not belong to $W$, then by Lemma 1 , it is easy to see that $W$ becomes $\gamma_{t}^{L}$-set for $U$ and

$$
\gamma_{t}^{L}(U)=|W|=\gamma_{t}^{L}(\Im) \leq \frac{r+l+2}{2} .
$$

Now suppose $u \notin W, v \in W$, and $v$ is a pendant vertex. Then there exists support vertex $s$ such that $v \sim s$. By Proposition $1, s \in W$. Now as $v$ is a pendant, $N(v) \cap W=\{s\}$ in $\mathfrak{J}$, and $v$ is not adjacent to any vertex of $V(T)-W$. Also, $u$ is adjacent to some vertex $v \neq w \in W$ so that in $U,\{w, v\} \subseteq N(u) \cap W$. As in $U, v$ is not adjacent to any other vertex of $V(U)-W$ except $u$, so $N(u) \cap W \neq N(y) \cap W$ for all $y \in V(U)-W$. Moreover, for all $u \neq y, z \in$ $V(U)-W, N(y) \cap W \neq N(z) \cap W$ because for all $u \neq y$, $z \in V(T)-W, N(y) \cap W$ and $N(z) \cap W$ remain the same in both $\mathfrak{I}$ and $U$. This further implies that, in this case, $W$ again becomes $\gamma_{t}^{L}$-set for $U$. Therefore, by Theorem 1 and the fact that either $l=l^{\prime}-1$ or $l=l^{\prime}-2$, we have

$$
\gamma_{t}^{L}(U)=|W|=\gamma_{t}^{L}(\mathfrak{I}) \leq \frac{r+l^{\prime}}{2} \leq \frac{r+l+2}{2} .
$$

Finally, suppose that all $u \in W, v \notin W$, and $v$ is not a leaf. Then, $W \cup\{v\}$ is $L T D$-set for $U$. Therefore, by Theorem 1 , we have

$$
\gamma_{t}^{L}(U) \leq|W|+1=\gamma_{t}^{L}(\Im)+1 \leq \frac{r+l^{\prime}}{2}+1 .
$$

If $u$ is a pendant vertex, then $l=l^{\prime}-1$ and by (10), we have

$$
\gamma_{t}^{L}(U) \leq \frac{r+l+1}{2}+1=\frac{r+l+3}{2} .
$$

But if $u$ is not a pendant vertex, then $l^{\prime}=l$ and by (10)

$$
\gamma_{t}^{L}(U) \leq \frac{r+l}{2}+1=\frac{r+l+2}{2} .
$$

The result follows from (8), (9), (11), and (12).

If in a unicycle graph $U$ with cycle length $m$, all the edges of its unique cycle are removed, then resulting graph is a disconnected graph which is union of $m$ disjoint subtrees $T_{i}$, where $i=1, \ldots \ldots, m$. Each subtree $T_{i}$ is a maximal subtree of $U$ containing only one cycle vertex. A bicyclic graph $B$ can 
be constructed from a noncorona unicyclic graph $U$ by choosing any two nonadjacent vertices $u, v \in V\left(T_{i}\right) \subseteq V(U)$ for some $i$ (i.e., $u$ and $v$ must be in one subtree $T_{i}$ of $U$ ) and joining the vertices must contain at least one cycle edge, $u$ and $v$ through an edge. It is easy to see that any path between vertices of two distinct subtrees.

Lemma 2. Consider a unicyclic graph $U$ with $r>5$ vertices and $l^{\prime}$ leaves and $\gamma_{t}^{L}$-set $W^{\prime}$. Choose any two nonadjacent vertices $u$ and $v$ from maximal subtree Ti of $U$ containing exactly one cycle vertex such that either both belong to $W^{\prime}$ or none of them belongs to $W^{\prime}$. Let $B$ be a bicyclic graph having $r>5$ vertices and $l$ pendant vertices obtained by connecting $u, v \in V(U)$ through an edge. Then,

$$
\gamma_{t}^{L}(B) \leq \frac{r+l+5}{2} .
$$

Proof. The proof is the same as that of Lemma 1.

Theorem 3. For bicyclic graph $B$ with order $r>4$ and $l$ pendant vertices, one has

$$
\gamma_{t}^{L}(B) \leq \frac{r+l+6}{2} .
$$

Proof. Let $U$ be the unicyclic graph of order $r>4$ with $l^{\prime}$ pendant vertices and $u, v \in V\left(T_{i}\right) \subseteq V(U)$ be its two nonadjacent vertices, where $T_{i}$ is maximal subtree of $U$ containing exactly one cycle vertex. Then, bicyclic graph $B$ can be obtained from $U$ by joining $u$ and $v$. Let $W^{\prime}$ be a $\gamma_{t}^{L}$-set of $U$. Now the vertices $u$ and $v$ may belong to $W^{\prime}$ or not.

The result follows by using Lemma 2 and the similar arguments as used in Theorem 2.

Now, as an extension of Theorems 2 and 3, an upper bound for locating-total domination number of cacti graphs is appraised as follows.
Theorem 4. Let $\zeta$ be cacti with $r$ vertices, 1 pendant vertices, and $q$ cycles. Then, one has

$$
\gamma_{t}^{L}(\zeta) \leq \frac{r+l+3 q}{2}
$$

Proof. We prove this by making use of mathematical induction on $q$. If $q=1,2$, then the desired result follows immediately by Theorems 2 and 3. Hence, we have a base for induction. Now assume that the bound is satisfied for any cacti graph having order $r$ and containing $q-1$ cycles. Now a cactus $\zeta$ with order $r$, leaves $l$, and cycles $q$ can be obtained from a cactus $\zeta^{\prime}$ of order $r$ with $l^{\prime}$ leaves, $q-1$ cycles, and $\gamma_{t}^{L}$-set $W$ in one of the following three ways:

Select any two pendant vertices $u$ and $v$ of $\zeta^{\prime}$ such that the shortest path between $u$ and $v$ must not have any cycle edge. Now if the vertices $u$ and $v$ are connected by an edge, we get a cactus $\zeta$. In this case, we can see that the set $W$ is also $L T D$-set of $\zeta$ by using similar arguments as used in Theorems 2 and 3. Now here $l^{\prime}=l+2$ and using induction hypothesis, we have

$$
\begin{aligned}
\gamma_{t}^{L}(\zeta) & \leq \gamma_{t}^{L}\left(\zeta^{\prime}\right) \leq \frac{r+l^{\prime}+3(q-1)}{2}=\frac{r+l+2+3 q-3}{2} \\
& <\frac{r+l+3 q}{2} .
\end{aligned}
$$

Select a pendant vertex $u$ and a nonpendant vertex $v$ of $\zeta^{\prime}$ such that the shortest path between $u$ and $v$ does not include any cycle edge. Now if the vertices $u$ and $v$ are connected by an edge, we get a cactus $\zeta$. In the case, when $u \notin W, W \bigcup\{u\}$ is $L T D$-set of $\zeta$; otherwise, $W$ is $L T D$-set for $\zeta$ by using similar arguments as used in Theorems 2 and 3. Now here $l^{\prime}=l+2$ and using induction hypothesis, we have

$$
\gamma_{t}^{L}(\zeta) \leq \gamma_{t}^{L}\left(\zeta^{\prime}\right)+1 \leq \frac{r+l^{\prime}+3(q-1)}{2}+1=\frac{r+l+2+3 q-3+2}{2}=\frac{r+l+3 q}{2}
$$

Select any two nonpendant vertices $u$ and $v$ of $\zeta$ I such that the path between $u$ and $v$ does not include any cycle edge. Now if the vertices $u$ and $v$ are connected by an edge, we get a cactus $\zeta$. In the case, when exactly one of the $u$ or $v$, say $u$, belongs to $W$, then $W \cup\{v\}$ is $L T D$ - set; otherwise, $W$ is $L T D$-set for $\zeta$ by using similar arguments as used in Theorems 2 and 3. Now here $l^{\prime}=l$ and using induction hypothesis, we have

$$
\gamma_{t}^{L}(\zeta) \leq \gamma_{t}^{L}\left(\zeta^{\prime}\right)+1 \leq \frac{r+l^{\prime}+3(q-1)}{2}+1=\frac{r+l+2+3 q-3+2}{2}<\frac{r+l+3 q}{2}
$$


Hence, the result follows.

\section{Tadpole and Rooted Product Graphs}

In this section, locating-total domination number for few cacti graphs is determined. In particular, tadpole graphs (unicyclic graphs), rooted product of cycle by path (unicyclic graphs), and rooted product of $P_{q}$ by $C_{m}$ (cacti graph with $q$ cycles) are investigated.

Theorem 5 (see [1]). For $q \geq 3, \gamma_{t}^{L}\left(P_{q}\right)=\lfloor q / 2\rfloor+\lceil q / 4\rceil-$ $\lfloor q / 4\rfloor$.

Theorem 6 (see [4]). For $q \geq 3, \gamma_{t}^{L}\left(C_{q}\right)=\lfloor q / 2\rfloor+\lceil q / 4\rceil-$ $\lfloor q / 4\rfloor$.

Remark 1. The locating-total domination number of $C_{q}$ and $P_{q}$ for different values of $q$ can be simplified as

(1) $\gamma_{t}^{L}\left(C_{q}\right)=\gamma_{t}^{L}\left(P_{q}\right)=q / 2$ if $q \equiv 0(\bmod 4)$.

(2) $\gamma_{t}^{L}\left(C_{q}\right)=\gamma_{t}^{L}\left(P_{q}\right)=q / 2+1$ if $q \equiv 2(\bmod 4)$.

(3) $\gamma_{t}^{L}\left(C_{q}\right)=\gamma_{t}^{L}\left(P_{q}\right)=q+1 / 2$ if $q$ is odd.

Let $T_{q_{1}, q_{2}}^{r}(q \geq 3)$ be a tadpole having $r=q_{1}+q_{2}$ vertices and is formed by connecting a vertex of cycle $C_{q_{1}}$ to pendant vertex of path by an edge.

Theorem 7. Let $q_{1}$ be odd positive integer. Then, one has

$$
\gamma_{t}^{L}\left(T_{q_{1}, q_{2}}^{r}\right)= \begin{cases}\frac{r}{2}, & \text { if } q_{2} \equiv 1(\bmod 4), \\ \frac{r+1}{2}, & \text { if } q_{2} \equiv 0,2(\bmod 4), \\ \frac{r}{2}+1, & \text { if } q_{2} \equiv 3(\bmod 4), q_{1} \equiv 3(\bmod ) 4, \\ \frac{r}{2}, & \text { if } q_{2} \equiv 3(\bmod 4), q_{1} \equiv 1(\bmod ) 4 .\end{cases}
$$

Proof. Suppose that $P_{q_{2}}: a_{1} \longrightarrow a_{2} \longrightarrow a_{3} \longrightarrow \cdots \longrightarrow a_{\mathrm{q} 2}$ is connected through $a_{q_{2}}$ to a cycle vertex $u$ of $T_{q_{1}, q_{2}}^{r}$. Let $W$ be a $\gamma_{t}^{L}$-set for $T_{q_{1}, q_{2}}^{r}$. As $|W|$ is minimum, therefore $a_{2}$ being support vertex by Proposition 1 and $a_{3}$ being its unique neighbouring vertex by definition of locating-total dominating set must belong to $W$. Consequently, $a_{4}$ and $a_{5}$ can be excluded from $W$ and then the next two consecutive vertices belong to $W$. By continuing this process, we can see that for $j=2,3(\bmod 4), a \in W$ and for $j=0,1(\bmod 4), a_{j} \notin W$.

Case 1: Suppose $q_{2} \equiv 1(\bmod 4)$. If $q_{2}=1$, then $v_{q_{2}} \in T_{q_{1}, q_{2}}^{r}$ is a pendant vertex and $u$ is a support vertex. Thus, $v_{q_{2}}, u \in W$ and

$$
\gamma_{t}^{L}\left(T_{q_{1}, 1}^{r}\right)=\gamma_{t}^{L}\left(C_{q_{1}}\right)=\frac{r}{2}
$$

But if $q_{2}>1$, then as $q_{2} \equiv 1(\bmod 4), a_{q_{2}}, a_{q_{2}-1} \notin W$ and $a_{q_{2}-2}, a_{q_{2}-3} \in W$.

Consequently, total $\left(q_{2}-1\right) / 2$ vertices of $P_{q_{2}}$ belong to $W$. Since $a_{q_{2}}, a_{q_{2}-1} \notin W$, therefore $u \in W$. Thus, we have

$$
\gamma_{t}^{L}\left(T_{q_{1}, q_{2}}^{r}\right)=\gamma_{t}^{L}\left(C_{q_{2}}\right)+\frac{q_{2}-1}{2}=\frac{r}{2} .
$$

The $\gamma_{t}^{L}$-set of tadpole graph $T_{q_{1}, q_{2}}^{r}$ for $q \equiv 1(\bmod 4)$ is represented by black vertices in Figure 1 .

Case 2: Suppose $q_{2} \equiv 2(\bmod 4)$. If $q_{2}=2$, then $a_{q_{2}}$ is a support vertex. Therefore, $a_{q_{2}} \in W$. Also, using the definition of $L T D$-set, $u \in W$. This implies that $\left(q_{1}+\right.$ 1)/2 cycle vertices must belong to $W$ and hence we have

$$
\gamma_{t}^{L}\left(T_{q_{1}, 2}^{r}\right)=\frac{q_{1}+1}{2}+1=\frac{r+1}{2} .
$$

But if $q_{2}>2$, then as $q_{2} \equiv 2(\bmod 4)$, therefore $a_{q_{2}} \in D$ and $a_{q_{2}-1}, a_{q_{2}-2} \notin W$. Consequently, $q_{2} / 2$ path vertices belong to $W$. Also, since $a_{q_{2}} \in W$ and $a_{q_{2}-1}, a_{q_{2}-2} \notin W$, therefore $u \in W$ which implies that $\left(q_{1}+1\right) / 2$ cycle vertices must be in $W$. Hence,

$$
\gamma_{t}^{L}\left(T_{q_{1}, q_{2}}^{r}\right)=\frac{q_{1}+1}{2}+\frac{q_{2}}{2}=\frac{r+1}{2} .
$$

The $\gamma_{t}^{L}$-set of tadpole graph $T_{q_{1}, q_{2}}^{r}$ for $q \equiv 2(\bmod 4)$ is represented by black vertices as shown in Figure 2 .

Case 3: Now assume $q_{2} \equiv 0(\bmod 4)$. If $q_{2}=0$, then we have

$$
\gamma_{t}^{L}\left(T_{q_{1}, 0}^{r}\right)=\gamma_{t}^{L}\left(C_{q_{1}}\right)=\frac{r+1}{2}
$$

But if $q_{2}>0$, then as $q \equiv 0(\bmod 4)$ and $|W|$ is minimum, so $a_{q_{1}} \notin W$ and $a_{q_{2}-1}, a_{q_{2}-2} \in W$. Consequently, $q_{2} / 2$ path vertices lie in $W$. Also, since $a_{q_{2}} \notin W$ and $a_{q_{2}-1}$, $a_{q_{2}-2} \in W$ and $u$ can be excluded from $W$. This implies that $\left(q_{1}+1\right) / 2$ cycle vertices must lie in $W$ and hence

$$
\gamma_{t}^{L}\left(T_{q_{1}, q_{2}}^{r}\right)=\frac{q_{1}+1}{2}+\frac{q_{2}}{2}=\frac{r+1}{2} .
$$

The $\gamma_{t}^{L}$-set of tadpole $T_{q_{1}, q_{2}}^{r}$ for $q_{2} \equiv 0(\bmod 4)$ is shown in Figure 3 with black vertices.

Case 4: Finally, assume that $q_{2} \equiv 3(\bmod 4)$. If $q_{2}=3$, then $a_{2}$ being support vertex and $a_{3}$ being its neighbouring vertex lie in $W$. But then $u$ can be taken out of $W$. Moreover, for $q_{1} \equiv 3(\bmod 4),\left(q_{1}+1\right) / 2$ cycle vertices lie in $W$ and hence 


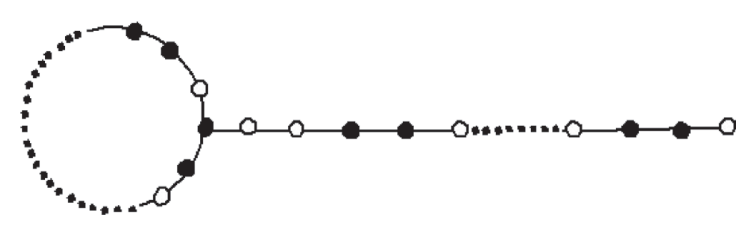

Figure 1: Tadpole $T_{q_{1}, q_{2}}^{r}$ for $q_{2} \equiv 1(\bmod 4)$.

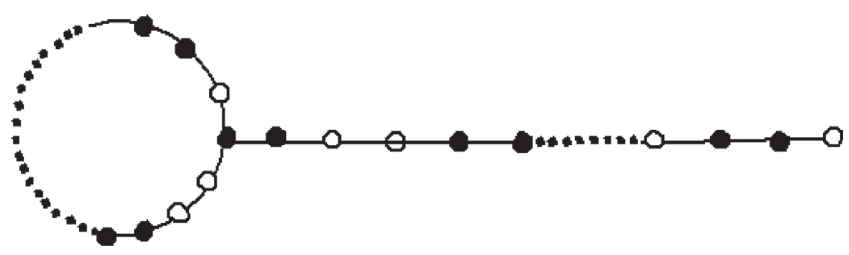

FIgURE 2: Tadpole $T_{q_{1}, q_{2}}^{r}$ for $q \equiv 2(\bmod 4)$.

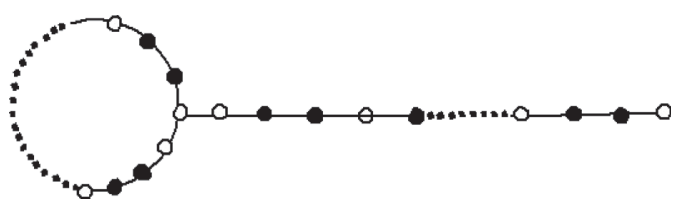

FIgure 3: Tadpole $T_{q_{1}, q_{2}}^{r}$ for $q_{2} \equiv 0(\bmod 4)$.

$$
\gamma_{t}^{L}\left(T_{q_{1}, 3}^{r}\right)=\frac{q_{1}+1}{2}+2=\frac{r}{2}+1
$$

On the other hand, if $q_{1} \equiv 1(\bmod 4)$, then $\left(q_{1}-1\right) / 2$ cycle vertices belong to $W$ and we have

$$
\gamma_{t}^{L}\left(T_{q_{1}, 3}^{r}\right)=\frac{q_{1}-1}{2}+2=\frac{r}{2} .
$$

Now if $q_{2}>3$, then as $q_{2} \equiv 3(\bmod 4)$; therefore, $a_{q_{2}}$, $a_{q_{2}-1} \in W$ and $a_{q_{2}-2}, a_{q_{2}-3} \notin W$. Consequently, $\left(q_{2}+1\right) / 2$ path vertices lie in $W$. Also, as $a_{q_{2}}, a_{q_{2}-1} \in W$, and $u$ can be excluded from $W$. But then the vertices which lie in $\gamma_{t}^{L}\left(C_{q_{1}}\right)$ must lie in $W$. Moreover, if $q_{1} \equiv 3(\bmod 4)$, then $\left(q_{1}+1\right) / 2$ cycle vertices are in $W$. Thus, we have

$$
\gamma_{t}^{L}\left(T_{q_{1}, q_{2}}^{r}\right)=\frac{q_{1}+1}{2}+\frac{q_{2}+1}{2}=\frac{r}{2}+1,
$$

whereas if $q_{1} \equiv 1(\bmod 4)$, then $\left(q_{1}-1\right) / 2$ cycle vertices belong to $W$ and we have

$$
\gamma_{t}^{L}\left(T_{q_{1}, q_{2}}^{r}\right)=\frac{q_{1}-1}{2}+\frac{q_{2}+1}{2}=\frac{r}{2} .
$$

The $\gamma_{t}^{L}$-set of tadpole $T_{q_{1}, q_{2}}^{r}$ for $q_{2} \equiv 3(\bmod 4)$ is shown in Figure 4 with black vertices.

Theorem 8. Let $q_{1}$ be an even integer. Then one has

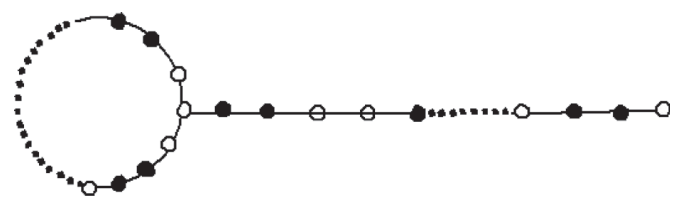

FIgURE 4: Tadpole $T_{q_{1}, q_{2}}^{r}$ for $q_{2} \equiv 3(\bmod 4)$.

$$
\gamma_{t}^{L}\left(T_{q_{1}, q_{2}}^{r}\right)= \begin{cases}\frac{r+1}{2}, & \text { if } q_{2} \equiv 1,3(\bmod 4), \\ \frac{r}{2}, & \text { if } q_{2} \equiv 2(\bmod 4), \\ \frac{r}{2}, & \text { if } q_{2} \equiv 0(\bmod 4), q_{1} \equiv 0(\bmod ) 4 \\ \frac{r}{2}+1, & \text { if } q_{2} \equiv 0(\bmod 4), q_{1} \equiv 2(\bmod ) 4 .\end{cases}
$$

Proof. Let $W$ be a $\gamma_{t}^{L}$-set for $T_{q_{1}, q_{2}}^{r}$. As $|\mathrm{W}|$ is minimum, therefore $a_{2}$ being support vertex by Proposition 1 and $a_{3}$ being its unique neighbouring vertex by definition of locating-total dominating set must belong to $W$. Consequently, $a_{4}$ and $a_{5}$ can be excluded from $W$ and then the next two consecutive vertices belong to $W$. By continuing this process, we can see that for $j \equiv 2,3(\bmod 4), a_{j} \in W$ and for $j \equiv 0,1(\bmod 4), a_{j} \notin W$.

Case 1: Suppose $q_{2} \equiv 1(\bmod 4)$. If $q_{2}=1$, then $a_{q_{2}} \in T_{q_{1}, q_{2}}^{r}$ is a pendant vertex and $u$ is a support vertex. Thus, $a_{q_{2}}, u \in W$ and

$$
\gamma_{t}^{L}\left(T_{q_{1}, 1}^{r}\right)=\frac{q_{1}}{2}+1=\frac{r+1}{2} .
$$

The $\gamma_{t}^{L}$-sets of tadpoles $T_{4,1}^{5}$ and $T_{6,1}^{7}$ are shown in Figure 5 with black vertices. But if $q_{2}>1$.

Then as $q_{2} \equiv 1 \quad(\bmod 4), \quad a_{q_{2}}, a_{q_{2}-1} \notin \quad \mathrm{W}$ and $a_{q_{2}-2}, a_{q_{2}-3} \in W$. Consequently, total $\left(q_{2}-1\right) / 2$ path vertices belong to $W$. Further, since $a_{q_{2}}, a_{q_{2}-1} \notin W$, therefore $u \in W$. Thus, $\left(q_{1} / 2\right)+1$ cycle vertices lie in $D$ and consequently,

$$
\gamma_{t}^{L}\left(T_{q_{1}, q_{2}}^{r}\right)=\frac{q_{1}}{2}+1+\frac{q_{2}-1}{2}=\frac{r+1}{2} .
$$

Case 2: Suppose $q_{2} \equiv 3(\bmod 4)$. If $q_{2}=3$, then $a_{2}$ being support vertex and $\mathrm{a}_{3}$ being its neighbouring vertex lie in $W$. And $u$ can be excluded from $W$. This implies that 


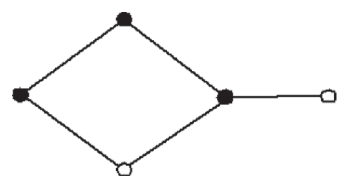

(a)

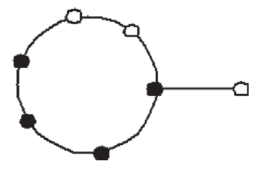

(b)
Figure 5: The tadpoles $T_{4,1}^{5}$ and $T_{6,1}^{7}$.

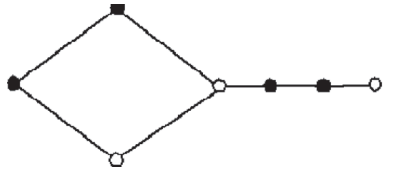

(a)

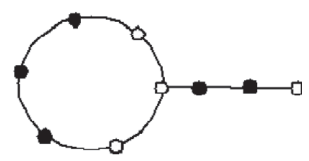

(b)
Figure 6: The tadpoles $T_{4,3}^{7}$ and $T_{6,3}^{9}$.

$q_{1} / 2$ cycle vertices must lie in $W$, and hence, we have

$$
\gamma_{t}^{L}\left(T_{q_{1}, 3}^{r}\right)=\frac{q_{1}}{2}+2=\frac{r+1}{2} .
$$

The $\gamma_{t}^{L}$-set of tadpoles $T_{4,3}^{7}$ and $T_{6,3}^{9}$ are shown in Figure 6 with black vertices.

But if $q_{2}>3$, then as $q_{2} \equiv 3(\bmod 4)$, so $a_{q_{2}}, a_{q_{2}-1}$ lie in $W$, whereas $a_{q_{2}-2}, a_{q_{2}-3} \notin W$. Consequently, $\left(q_{2}+1\right) / 2$ path vertices belong to $W$. Further, as $a_{q_{2}}, a_{q_{2}-1}$ lie in $W$ so $u$ can be taken out of $W$. Thus, $q_{1} / 2$ cycle vertices lie in $W$ so that

$$
\gamma_{t}^{L}\left(T_{q_{1}, q_{2}}^{r}\right)=\frac{q_{1}}{2}+\frac{q_{2}+1}{2}=\frac{r+1}{2} .
$$

Case 3: Now suppose $q_{2} \equiv 2(\bmod 4)$. If $q_{2}=2$, then $a_{q_{2}}$ being a support vertex belongs to $W$. This further implies that $u \in W$. Hence, $q_{1} / 2$ cycle vertices lie in $W$, and consequently,

$$
\gamma_{t}^{L}\left(T_{q_{1}, 2}^{r}\right)=\frac{q_{1}}{2}+1=\frac{r}{2}
$$

The $\gamma_{t}^{L}$-set of tadpoles $T_{4,2}^{6}$ and $T_{6,2}^{8}$ are shown in Figure 7 with black vertices.

On the other hand, if $q_{2}>2$, then $a_{q 2} \in W$ but $a_{q_{2}-1}$, $a_{q_{2}-2} \notin W$. This implies that $q_{2} / 2$ path vertices are included in $W$ along with cycle vertex $u$. Consequently, $q_{1} / 2$ cycle vertices lie in $W$ and we have

$$
\gamma_{t}^{L}\left(T_{q_{1}, q_{2}}^{r}\right)=\frac{q_{1}}{2}+\frac{q_{2}}{2}=\frac{r}{2} .
$$

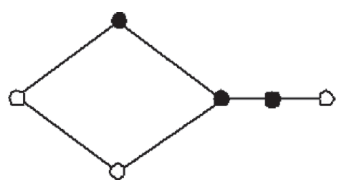

(a)

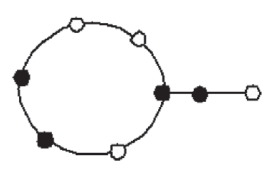

(b)
Figure 7: The tadpoles $T_{4,2}^{6}$ and $T_{6,2}^{8}$.

Case 4: Finally assume $q_{2} \equiv 0(\bmod 4)$. It is easy to see that if $q_{2}=0$, then $T_{q_{1}, 0}^{r} \cong C_{q_{1}}$ and $r=q_{1}$.

Hence,

$$
\gamma_{t}^{L}\left(T_{q_{1}, 0}^{r}\right)=\gamma_{t}^{L}\left(C_{q_{1}}\right)
$$

Using Remark 3, we have

$$
\gamma_{t}^{L}\left(T_{q_{1}, 0}^{r}\right)= \begin{cases}\frac{q_{1}}{2}, & \text { if } q_{1} \equiv 0(\bmod 4), \\ \frac{q_{1}}{2}+1, & \text { if } q_{1} \equiv 2(\bmod 4) .\end{cases}
$$

As $r=q_{1}$, so we have

$$
\gamma_{t}^{L}\left(T_{q_{1}, 0}^{r}\right)= \begin{cases}\frac{r}{2}, & \text { if } q_{1} \equiv 0(\bmod 4), \\ \frac{r}{2}+1, & \text { if } q_{1} \equiv 2(\bmod 4) .\end{cases}
$$

But if $q_{2}>0$, then $a_{q_{2}} \notin W$ and $a_{q_{2}-1}, a_{q_{2}-2} \in W$. This shows that $q_{2} / 2$ path vertices belong to $W$. Also, since $a_{q_{2}} \notin W$ and $a_{q_{2}-1}, a_{q_{2}-2} \in W, u$ must be in $W$. Hence, the vertices that belong to $\gamma_{t}^{L}\left(C_{q_{1}}\right)$ must lie in $W$. Hence, we have

$$
\gamma_{t}^{L}\left(T_{q_{1}, q_{2}}^{r}\right)=\frac{q_{2}}{2}+\gamma_{t}^{L}\left(C_{q_{1}}\right)
$$

Again, using Remark 3, we have

$$
\gamma_{t}^{L}\left(T_{q_{1}, q_{2}}^{r}\right)= \begin{cases}\frac{q_{2}}{2}+\frac{q_{1}}{2}, & \text { if } q_{1} \equiv 0(\bmod 4), \\ \frac{q_{2}}{2}+\frac{q_{1}}{2}+1, & \text { if } q_{1} \equiv 2(\bmod 4) .\end{cases}
$$

Thus,

$$
\gamma_{t}^{L}\left(T_{q_{1}, q_{2}}^{r}\right)= \begin{cases}\frac{r}{2}, & \text { if } q_{1} \equiv 0(\bmod 4), \\ \frac{r}{2}+1, & \text { if } q_{1} \equiv 2(\bmod 4) .\end{cases}
$$

In Theorem 9, the rooted product $P_{q_{2}} \odot C_{q_{1}}$ is computed. Any cycle vertex can be considered as root vertex.

Theorem 9. For positive integers $q_{1} \geq 3$ and $q_{2} \geq 1$, one has 


$$
\gamma_{t}^{L}\left(P_{q_{2}} \odot C_{q_{1}}\right)= \begin{cases}\gamma_{t}^{L}\left(C_{q_{1}}\right), & \text { if } q_{2}=1, \\ q_{2} \gamma_{t}^{L}\left(C_{q_{1}}\right), & \text { if } q_{1} \equiv 0,3(\bmod 4), \\ q_{2} \gamma_{t}^{L}\left(C_{q_{1}-1}\right)+\frac{q_{2}}{2}, & \text { if } q_{1} \equiv 1(\bmod 4), \\ q_{2} \gamma_{t}^{L}\left(C_{q_{1}-2}\right)+q_{2}, & \text { if } q_{1} \equiv 2(\bmod 4) .\end{cases}
$$

Proof. If $q_{2}=1$, then $\quad P_{q_{2}} \odot C_{q_{1}} \cong C_{q_{1}} \quad$ and $\gamma_{t}^{L}\left(P_{q_{2}} \odot C_{q_{1}}\right)=\gamma_{t}^{L}\left(C_{q_{1}}\right)$. Now we may suppose $q_{2} \geq 2$.

Then four cases arise:

Case 1: Suppose $q_{1} \equiv 0(\bmod 4)$, that is, $q_{1}=4 k$ for integer $k>0$. The graph $P_{q_{2}} \odot C_{q_{1}}$ contains $q_{2}$ mutually disjoint cycles $C_{q_{1}}^{i}$, where $i=1, \ldots, q_{2}$. For each $i$, cycle $C_{q_{1}}^{i}$ consists of $4 k$ vertices, namely, $v_{j}^{i}$ where $j=1, \ldots$, $4 k$. Take $v_{1}^{i}$ as root vertex of each cycle. The $4 k$ vertices in each cycle are divided into $k$ sets such that each set contains four consecutive vertices of cycle. From each set, the middle two vertices can be taken as elements of minimum LTD-set for each $C_{m}^{i}$. Let this minimum LTD-set of $C_{m}^{i}$. Then $W_{i}=\left\{v_{2}^{i}, v_{3}^{i}, v_{6}^{i}, v_{7}^{i}\right.$, $\left.\ldots, v_{4 k-2}^{i}, v_{4 k-1}^{i}\right\}$ with $\left|W_{i}\right|=2 k=\gamma_{t}^{L}\left(C_{m}^{i}\right)$. These $2 k$ vertices in each $W_{i}$ are shown in Figure 8 as black vertices.

Now the root vertices $v_{1}^{i}$ from each $C_{m}^{i}$ are actually the path vertices and are locating totally dominated by the elements of $W_{i}$ for each $i$. Hence, all the vertices of $P_{q_{2}} \odot C_{q_{1}}$ are locally dominated by $\cup_{i=1}^{q_{2}} W_{i}$. Also, this set is minimum by construction. Thus,

$$
\gamma_{t}^{L}\left(P_{q_{2}} \odot C_{q_{1}}\right)=q_{2} \gamma_{t}^{L}\left(C_{q_{1}}\right)
$$

Case 2: Suppose $q_{1} \equiv 1(\bmod 4)$, that is, $q_{1}=4 k+1$, for integer $k>0$. Let $v_{1}^{i}$ be the root vertex of cycle $C_{q_{1}}^{i}$ for each $i$. The graph $C_{q_{1}}^{1}-\left\{v_{1}^{1}\right\}$ contains $4 k$ vertices and these $4 k$ vertices are divided into $k$ sets. Now we select two middle vertices from each set. Then, all $4 k$ vertices of $C_{q_{1}}^{1}-\left\{v_{1}^{1}\right\}$ are locating totally dominated by $2 k$ chosen vertices. Since $v_{1}^{1}$ does not have a neighbour in the selected LTD-set of $C_{q_{1}}^{1}$, therefore, $v_{1}^{2}$ must be a LTD-vertex. The graph $C_{q_{1}}^{2}-\left\{v_{1}^{2}\right\}$ also contains $4 k$ vertices and these $4 k$ vertices are again divided into $k$ sets and all are locating totally dominated by $2 k$ vertices by selecting the first and last vertices from each set. Now we are interested in minimum LTD-set; $v_{1}^{3}$ may not be an $L T D$-vertex. By continuing in this way, we can see that if $i$ is odd then $v_{1}^{i}$ is not an $L T D$-vertex otherwise $v_{1}^{i}$ is an LTD-vertex. This along with the fact that path vertices are root vertices implies that all the vertices of graph are locating totally dominated by a set $W$ of smallest cardinality. We note that if the path (root) vertex is not an LTD-vertex, then associated cycle has $2 k$ vertices in $W$ and otherwise each cycle has $2 k+1$ vertices in $W$. Hence,

$$
\gamma_{t}^{L}\left(P_{q_{2}} \odot C_{q_{1}}\right)=q_{2} \gamma_{t}^{L}\left(C_{q_{1}-1}\right)+\frac{q_{2}}{2} .
$$

The $\gamma_{t}^{L}$-set of $P_{q_{2}} \odot C_{q_{1}}$ for $q_{1} \equiv 1(\bmod 4)$ is shown in Figure 9 with black vertices.

Case 3: Suppose $q_{1} \equiv 2(\bmod 4)$ such that $q_{1}=4 k+2$ and $k>0$ is an integer. In a similar manner as in Cases 1 and 2 , we divide the vertices of each $C_{q_{1}}^{i}-\left\{v_{1}^{1}, a_{\mathrm{i}}\right\}$, where $a_{i}$ is neighbouring vertex of root vertex into $k$ sets. For each $i$, all the vertices of $C_{q_{1}}^{i}-\left\{v_{1}^{1}, a_{\mathrm{i}}\right\}$ are locating totally dominated by $2 k$ chosen vertices (middle two vertices in each set). The vertices $v_{1}^{i}$ and $a_{i}$ for all $i$ can be locating totally dominated if we include all the root vertices. This implies that

$$
\gamma_{t}^{L}\left(P_{q_{2}} \odot C_{q_{1}}\right)=q_{2} \gamma_{t}^{L}\left(C_{q_{1}-2}\right)+q_{2} .
$$

The $\gamma_{t}^{L}$-set of $P_{q_{2}} \odot C_{q_{1}}$ for $q_{1} \equiv 2(\bmod 4)$ is shown in Figure 10 with black vertices.

Case 4: Finally, suppose $q_{1}=4 k+3$, where $k>0$ is an integer. Here, the vertices of $C_{q_{1}}^{i}-\left\{v_{1}^{1}, a_{i}, b_{i}\right\}$, where $a_{i}$ and $b_{i}$ are consecutive two vertices of root vertex $v_{1}^{i}$ on cycle, are divided into $k$ sets. Again for each $i$, all the vertices $C_{q_{1}}^{i}-\left\{v_{1}^{1}, a_{\mathrm{i}}, b_{\mathrm{i}}\right\}$ are locating totally dominated by $2 k$ chosen vertices (first two vertices in each set). The vertices $\left\{v_{1}^{1}, a_{i}, b_{i}\right\}$ for all $i$ can be locating-total dominated by selecting two of them except root vertex. Therefore, in this case, we have

$$
\gamma_{t}^{L}\left(P_{q_{2}} \odot C_{q_{1}}\right)=q_{2} \gamma_{t}^{L}\left(C_{q_{1}}\right)
$$

The $\gamma_{t}^{L}$-set of $P_{q_{2}} \odot C_{q_{1}}$ for $q_{1} \equiv 3(\bmod 4)$ is shown in Figure 11 with black vertices.

In the following Theorem 10, the rooted product $C_{q_{1}} \odot P_{q_{2}}$ is computed. Here, we select pendant vertex of path as root vertex.

Theorem 10. For $q_{1} \geq 3$ and $q_{2} \geq 2$ and by taking pendant vertex of $P_{q_{2}}$ as root vertex, one has

$$
\gamma_{t}^{L}\left(C_{q_{1}} \odot P_{q_{2}}\right)= \begin{cases}q_{1} \gamma_{t}^{L}\left(P_{q_{2}}\right), & \text { if } q_{2} \equiv 0,2(\bmod 4), \\ q_{1} \gamma_{t}^{L}\left(P_{q_{2}-1}\right)+\gamma_{t}^{L}\left(C_{q_{1}}\right), & \text { if } q_{2} \equiv 1(\bmod 4), \\ q_{1} \gamma_{t}^{L}\left(P_{q_{2}-3}\right)+2 q_{1}, & \text { if } q_{2} \equiv 3(\bmod 4) .\end{cases}
$$

Proof

Case 1: Suppose $q_{2} \equiv 0(\bmod 4)$, such that $q_{2}=4 k$ and $k>0$. The rooted product $C_{q_{1}} \odot P_{q_{2}}$ consists of mutually disjoint $q_{1}$ paths $P_{q_{2}}^{i}$ for $i=1, \ldots, q_{1}$. Suppose $v_{1}^{i}$ is the 


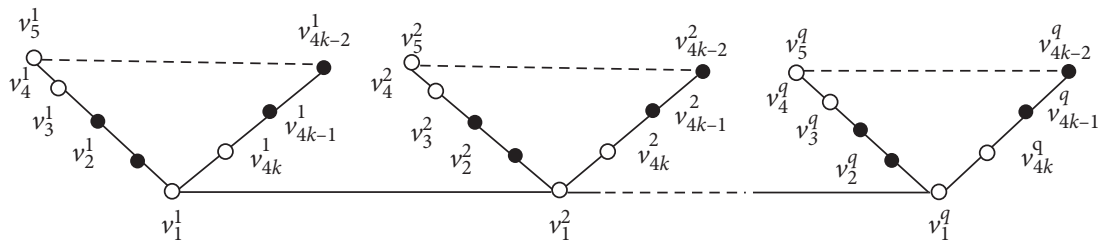

FIgURE 8: The rooted product $P_{q_{2}} \odot C_{q_{1}}$ for $q_{1} \equiv 0(\bmod 4)$.

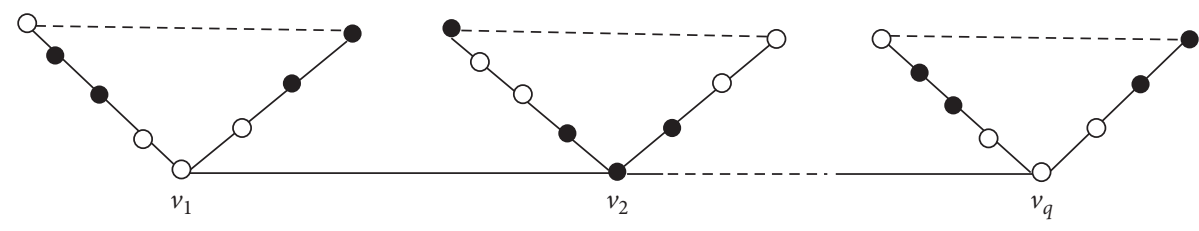

FIGURE 9: The rooted graph $P_{q_{2}} \odot C_{q_{1}}$ for $q_{1} \equiv 1(\bmod 4)$.

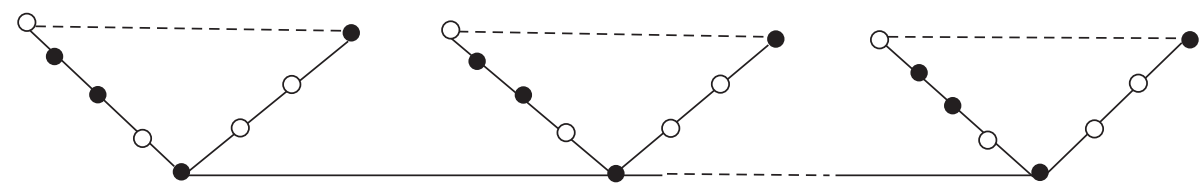

Figure 10: The graph $P_{q_{2}} \odot C_{q_{1}}$ for $q_{1} \equiv 2(\bmod 4)$.

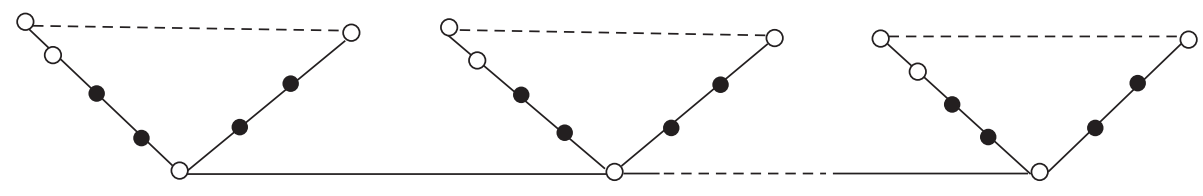

Figure 11: The graph $P_{q_{2}} \odot C_{q_{1}}$ for $q_{1} \equiv 3(\bmod 4)$.

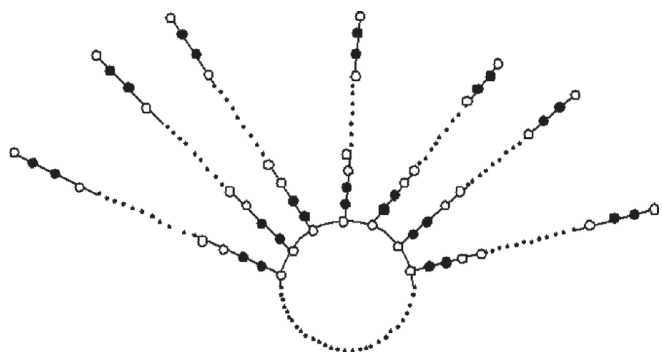

Figure 12: The product $C_{q_{1}} \odot P_{q_{2}}$ for $q_{2} \equiv 0(\bmod 4)$.

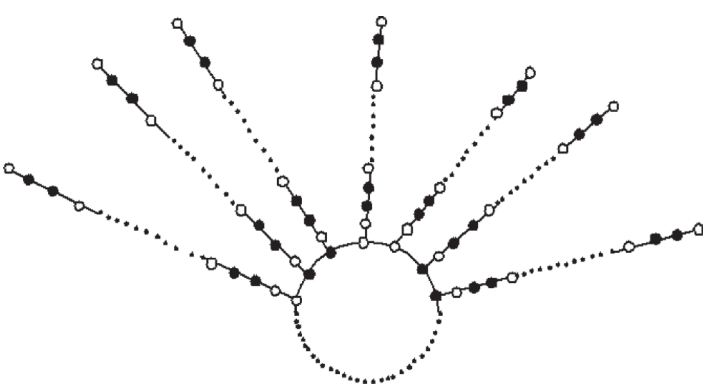

Figure 13: The graph $C_{q_{1}} \odot P_{q_{2}}$ for $q_{2} \equiv 1(\bmod 4)$. 


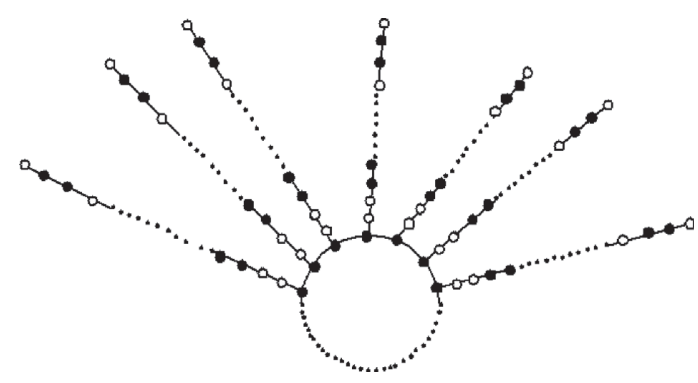

FIgURE 14: The product $C_{q_{1}} \odot P_{q_{2}}$ for $q_{2} \equiv 2(\bmod 4)$.

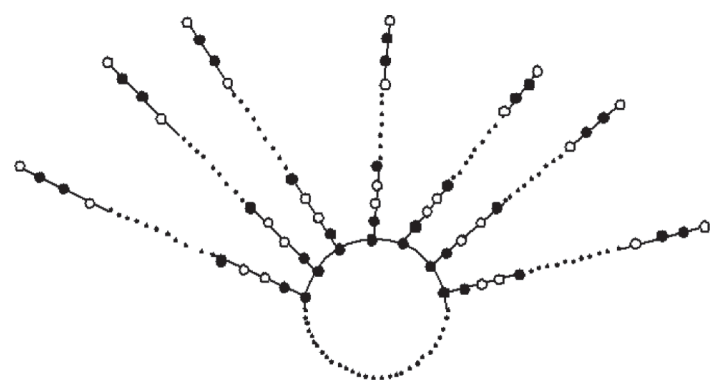

Figure 15: The graph $C_{q_{1}} \odot P_{q_{2}}$ for $q_{2} \equiv 3(\bmod 4)$.

root vertex of $P_{q_{2}}^{i}$ for $i=1, \ldots, q_{1}$. The $4 k$ vertices of $P_{q_{2}}^{i}$ are split into $k$ sets, each containing four consecutive vertices. Then the collection of middle two vertices from each set in every path gives the $L T D$-set for graph $C_{q_{1}} \odot P_{q_{2}}$ which is also minimum one:

$$
\gamma_{t}^{L}\left(C_{q_{1}} \odot P_{q_{2}}\right)=q_{1} \gamma_{t}^{L}\left(P_{q_{2}}\right)
$$

The $\gamma_{t}^{L}$-set of $C_{q_{1}} \odot P_{q_{2}}$ for $q_{2} \equiv 0(\bmod 4)$ is represented by black vertices in Figure 12 .

Case 2: Assume $q_{2} \equiv 1(\bmod 4)$ such that $q_{2}=4 k+1$ and $k>0$. Let $v_{o}^{i}$ be the root vertex in each path $P_{q_{2}}^{i}$ of $C_{q_{1}} \odot P_{q_{2}}$. Consider $P_{q_{2}}^{i}-\left\{v_{o}^{i}\right\}$ consisting of $4 k$ vertices and all the vertices in $P_{q_{2}}^{i}-\left\{v_{o}^{i}\right\}$ can be locating totally dominated by $2 k$ chosen vertices (two middle vertices from each set). Since all the paths $P_{q_{2}}^{i}$ for $i=1, \cdots, q_{1}$ are mutually disjoint, therefore $\cup_{i}^{q_{1}}\left\{P_{q_{2}}^{i}-\left\{v_{o}^{i}\right\}\right\}$ is minimum locating-total dominated set consisting of $2 k \cdot q_{1}$ vertices. Therefore,

$$
\begin{aligned}
\gamma_{t}^{L}\left(C_{q_{1}} \odot P_{q_{2}}\right) & =2 k q_{1}+\gamma_{t}^{L}\left(C_{q_{1}}\right) \\
& =q_{1} \gamma_{t}^{L}\left(P_{q_{2}-1}\right)+\gamma_{t}^{L}\left(C_{q_{1}}\right) .
\end{aligned}
$$

The $\gamma_{t}^{L}$-set of $C_{q_{1}} \odot P_{q_{2}}$ for $q_{2} \equiv 1(\bmod 4)$ is represented by black vertices as shown in Figure 13 .

Case 3: Now suppose that $q_{2} \equiv 2(\bmod 4)$ such that $q_{2}=4 k+2$. Here, each $P_{q_{2}}^{i}$ for $i=1, \cdots, q_{1}$ consists of $4 k+2$ vertices. All of them except the root and its neighbouring vertex are locating totally dominated by $2 k$ chosen vertices from each path as in the previous cases. These chosen vertices must belong to $\gamma_{t}^{L}$-set. To obtain, minimum $L T D$-se for rooted product, all the root vertices are also included. Hence,

$\gamma_{t}^{L}\left(C_{q_{1}} \odot P_{q_{2}}\right)=2 k q_{1}+q_{1}=q_{1} \gamma_{t}^{L}\left(P_{q_{2}-2}\right)+q_{1}$.

The $\gamma_{t}^{L}$-set of $C_{q_{1}} \odot P_{q_{2}}$ for $q_{2} \equiv 2(\bmod 4)$ is represented by black vertices in Figure 14 .

Case 4: The case $q_{2} \equiv 3(\bmod 4)$ is dealt in a similar manner as in previous cases. Thus, we have

$$
\gamma_{t}^{L}\left(C_{q_{1}} \odot P_{q_{2}}\right)=q_{1} \gamma_{t}^{L}\left(P_{q_{2}-3}\right)+2 q_{1} .
$$

The $\gamma_{t}^{L}$-set of $C_{q_{1}} \odot P_{q_{2}}$ for $q_{2} \equiv 3(\bmod 4)$ is shown in Figure 15 with black vertices.

\section{Conclusion}

In this paper, the upper bounds for locating-total domination number of unicyclic and bicyclic graphs are obtained. It is shown that, for unicyclic graph $\mathrm{U}$ of order $r$ and leaves $l$, $\gamma_{t}^{L}(U) \leq r+l+3 / 2$ and for bicyclic graph $\mathrm{B}$ of order $r$ and leaves $l$, $\gamma_{t}^{L}(B) \leq r+l+6 / 2$. These bounds are then generalized for cacti graphs of order $n$ having $q$ cycles as $r+l+3 q / 2$. Also, the exact value of locating-total domination numbers for some cacti graphs is determined. For tadpole graph $T_{q_{1}, q_{2}}^{r}$, it is found that locating-total dominating number is $r / 2, r+1 / 2$, or $r / 2+1$ depending upon the parity of $r$ and values of $q_{1}$ and $q_{2}$. The locating-total dominating number of rooted product of $C_{q 1}$ by $P_{q 2}$ in terms of $q_{1}$ and $\gamma_{t}^{L}\left(P_{q_{2}}\right)$ is computed. Similarly, locating-total dominating number of rooted products of $P_{q 2}$ by $C_{q 1}$ in terms of $q_{2}$ and $\gamma_{t}^{L}\left(C_{q_{1}}\right)$ is also determined. It is still an open problem to find the sharpness of estimated bounds of locating-total dominating number for cacti graphs.

\section{Data Availability}

All the required data are included within the paper. However, the reader may contact the corresponding author for more details of the data.

\section{Conflicts of Interest}

The authors do not have any conflicts of interest.

\section{Acknowledgments}

This work was sponsored by Shandong Provincial Natural Science Foundation, China (ZR2018MA010).

\section{References}

[1] T. W. Haynes, M. A. Henning, and J. Howard, "Locating and total dominating sets in trees," Discrete Applied Mathematics, vol. 154, no. 8, pp. 1293-1300, 2006. 
[2] X.-G. Chen and M. Y. Sohn, "Bounds on the locating-total domination number of a tree," Discrete Applied Mathematics, vol. 159, no. 8, pp. 769-773, 2011.

[3] M. Chellali and N. Jafari Rad, "Locating-total domination critical graphs," Australasian Journal of Combinatorics, vol. 45, pp. 227-234, 2009.

[4] M. A. Henning and C. Löwenstein, "Locating-total domination in claw-free cubic graphs," Discrete Mathematics, vol. 312, no. 21, pp. 3107-3116, 2012.

[5] J. McCoy and M. A. Henning, "Locating and paired-dominating sets in graphs," Discrete Applied Mathematics, vol. 157, no. 15, pp. 3268-3280, 2009.

[6] S. B. Rashmi and I. P. Kelkar, "Total domination number of rooted product graph," Journal of Computer and Mathematical Sciences, vol. 8, no. 6, pp. 0976-5697, 2017.

[7] H. Xing and M. Y. Sohn, "Bounds on locating total domination number of the Cartesian product of cycles and paths," Information Processing Letters, vol. 115, no. 12, pp. 950-956, 2015.

[8] W. Ning, M. Lu, and K. Wang, "Bounding the location-total domination number of a tree in terms of its annihilation number," Discussiones Mathematicae Graph Theory, vol. 39, no. $1,2019$.

[9] U. Ahmad and J. Ibrahim, "Lower bound of locating-total domination of unicyclic graphs," preprint. 